\title{
Activity Curvature: A new Approach to Perception
}

\author{
Konrad Weigl \\ INRIA B.P.93 06902 Sophia-Antipolis Cedex FRANCE \\ weigl@isophia.inria.ir
}

\section{Abstract}

We present a new approach to perception: The perception of objects by organisms is the deformation the objects cause to differentiable manifolds of sensory filters in function space; this deformation is characteristic for the manifold and the object perceived, and thus does depend on the organism and the outside stimulus. This warping by the stimulus can be considered as analogous to the warping caused by a large gravitational body to the four-dimensional spacetime manifold in its neighborhood.

We model a layer of the sensory cortex as a manifold in function space, e.g. a layer of simple cells of the primate visual cortex as a manifold of Gabor filters $g\left(x, \phi^{j}\right)[2]$, with a parameter vector $\phi^{j}$. The derivative vectors $\frac{\delta}{\delta \phi}$ form then a natural base for local tangent spaces to the manifold [3]. A stimulus $\mathrm{A}(\mathrm{x})$ entering through the pathways $\mathrm{x}$ generates an activity potential $f\left(x, \phi^{j}\right)=\left\langle A(x) \mid g\left(x, \phi^{j}\right)\right\rangle=\sum_{x=1}^{m} A(x) g\left(x, \phi^{j}\right)$ expressible in the local base of $\frac{\delta}{\delta \phi^{j}}$. We can thus define an activity metric for that manifold, via a metric tensor $g_{\mu \nu}$, analogous to the well-known Fisher Information Metric of the manifold of probabilities [1], but derived from $f\left(x, \phi^{j}\right)$ :

$$
g_{\mu \nu}=\sum_{x=1}^{m} \frac{\delta\left(A(x) g\left(x, \phi^{J}\right)\right)}{\delta \phi^{\mu}} \frac{\delta\left(A(x) g\left(x, \phi^{\nu}\right)\right)}{\delta \phi^{\nu}} \Rightarrow g_{\mu \nu}=\sum_{x=1}^{m} A(x)^{2} \frac{\delta f\left(x, \phi^{\nu}\right)}{\delta \phi^{\mu}} \frac{\delta f\left(x, \phi^{\nu}\right)}{\delta \phi^{\nu}}
$$

$A(x)$, through changing $f\left(x, \phi^{j}\right)$ from its resting state, changes the metric, and thus causes the activity curvature of the manifold to change: The local bases warp differently as we move along the intrinsic coordinate axes defined by the parameter vector. This warping/curving can be expressed by the Riemannian Curvature Tensor, computable as follows: For a given input $A(x)$, we obtain $g_{\mu \nu}$ through (1). The connection coefficients $\Gamma_{\alpha \beta}^{\mu}$ and the Riemannian Curvature Tensor $R_{\beta \gamma \tau}^{\alpha}$ will then be:

$$
\Gamma_{\alpha \beta}^{\mu}=\sum_{\nu} \frac{1}{2} g^{\mu \nu}\left(\frac{\delta g_{\nu \alpha}}{\delta \phi^{\beta}}+\frac{\delta g_{\nu \beta}}{\delta \phi^{\alpha}}-\frac{\delta g_{\alpha \beta}}{\delta \phi^{\nu}}\right) \quad R_{\beta \gamma \tau}^{\alpha}=\frac{\delta \Gamma_{\beta \tau}^{\alpha}}{\delta \phi^{\top}}-\frac{\delta \Gamma_{\beta \gamma}^{\alpha}}{\delta \phi^{\top}}+\sum_{\mu} \Gamma_{\mu \gamma}^{\alpha} \Gamma_{\beta \tau}^{\mu}-\sum_{\mu} \Gamma_{\mu \tau}^{\alpha} \Gamma_{\beta \gamma}^{\mu}
$$

As a simple example [4], we consider a manifold of gaussians, two-dimensional because we have two parameters, mean and standard deviation: We can now simply compute the coordinate independent scalar curvature $S c$ of such a manifold via the equations above as function of different inputs $A(x)$ and location on the manifold:

$$
S c=\sum_{\nu} \frac{g_{1 \nu} R_{212}^{\nu}}{g_{11} g_{22}-g_{12} g_{21}}
$$

Besides potential applications to model results obtained by brain recordings, we conjecture that the brain itself uses that curvature to perceive the environment, disregarding the reaction of individual neurons or clusters, instead integrating the reaction of the whole manifold/layer. One could conceive the brain measuring the curvature by measuring the relative arceleration of nearby geodesics, for example by waves travelling over the corlex, or measuring relative distances by comparing locally the neuronal activity.

We could then interpret habituation, learning, etc., as prewarping of the manifold.

Keywords: Biological Vision, Perception, Differential Geometry, Riemannian Spaces, Gabor functions.

[1] Caianiello, E.R., Systems and Uncertainty: A Gieometric Approach, in: Caianiello, E.R., Aizerman, M.A., Topics in the General Theory of Structures, D.Reidel, Publ., 1987

[2] Daugman, J., Six Formal Properties of Two-dimensional Anisotropic Visual Filters: Structural Principles and Frequency/Orientation Selectivity, IEEE trans. SNC, vol. SMC-13, Sept/Oct 1983 [3] Misner, C.W., Thorne, K.S., and Wheeler. J.A., Gravitation. Freeman \& Co., Pub., N.Y. 1970 [4] Weigl, Konrad, A new Approach to Perception: Activity Curvature, Tech. Rep. INRIA, to be published. 